\title{
Surface Plasmon Enhanced Transient Thermoreflectance
}

\author{
S. Herminghaus ${ }^{1, *}$ and P. Leiderer ${ }^{2}$ \\ 1 Institut für Physik, Universitäl Mainz, D-6500 Mainz, Feod. Rep. Germady \\ 2 Faxultäl für Physik, Universităt Konstanz, D-7750 Konstanz. Fed. Rep. Geronany \\ Recived 14 Decomber 1989/Acoepled 15 April 1990
}

\begin{abstract}
It is shown that optically excited surface plaswons can be used for measurements of the surface temperature of a metal with nanosecond time resolution. This method is closely related to transient thermoreflectance, but its sensitivity is considerably higher. We give a survey on the mechanisms involved, briefly discussing the dependence on the sample properties. Experimental confinmation of the proposed sensitivity enhancement as well as the time resolution is presented.
\end{abstract}

PACS: $07.60,42.80,61.80$

Transient thermoreflectance is a well established technique for observing temperature changes at a metal surface with high temporal resolution [1,2]. It employs as a probe the thermally induced change in the optical constants of the metal by measuring the intensity of a light beam being reflected at the surface under consideration. However, since the optical constants of the metal are not very sensitive to the temperature, signals obtained are usually small, and enbancement of the sensitivity of the method would be desirable. In this paper we demonstrate a modified transient thermoreflectance setup using an attenuated total reflectance technique. When the conditions for optical excitation of surface plasmons are fulfilled, the thermoreflectance sigual becomes resonantly enhanced, thus increasing the sensitivity of the measured light intensity to changes of the surface temperature. We elaborate on the feasibility of this effect for performing fast high sensitivity measurements of temperature changes at a metal surface.

\section{Theory}

Optical excitation of surface plasmons by means of the socalled Kretschmann setup has been described by many authors [3-5]: the sample is formed by a metal filon with a thickness of several tens of nanometers, evaporated onto the base of a glass prism. The suriace to be studied is the interface between the metal and the vacuum. On illu-

\footnotetext{
- Preseat address: IBM Almadea Research Conter, 650 Harty Road. San Jose, CA 95120 , USA
}

minating the film with a light beam coming through the prism at a certain angle of incidence, surface plasmons are resonantly excited by the evanescent wave on the metalvacuum interface. The measured quantity is the intensity of the reflected light, $I(\theta)$, which sbows a pronounced attenuation at the resonance angle due to conversion of pbotons into surface plasmons. The depth of this resonance dip depends strongly on the coupling between the surface plasmons and the photons, and thus it depends on the thickness of the metal film. For a given film material and light wavelength, there exists a certain film thickness which makes the reflected intensity drop to zero at the resonance angle. The resonance width depends on the film material; it is especially narrow on silver films, where it amounts to less than $0.5^{\circ}$.

When the film is being heated (e.g. with a laser pulse), the dielectric function of the film changes, affecting the position and width of the surface plasmon resonance. In order to discuss this detail, we first consider the temperature profile in the sample. Due to the high thermal conductivity of the metal film and its small thickness, it rapidly assumes a uniform temperature with respect to the normal direction of its surface. A silver film of about $50 \mathrm{~nm}$ thickness on a glass substrate, like the one used in this work, becomes uniform in temperature across its tbickness within less than a nanosecond, as shown recently [6]. Lateral temperature gradients can of course occur, for instance as a consequence of an inhomogeneous profile of the heating pulsed laser spot, on length scales large compared to the film thickness. When the ternperature profile in the film is uniform, so is the thermally induced change in the dielectric function, which therefore 


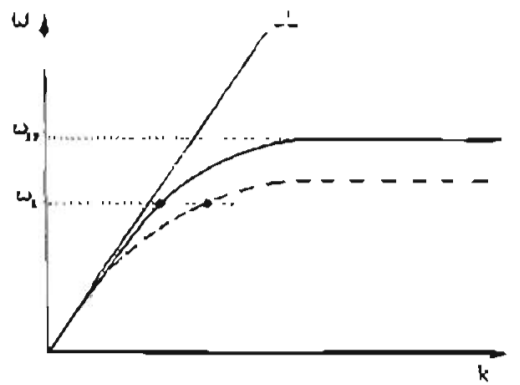

Fig. 1. Dispersion relation of the surface plasmons. For $k \rightarrow 0$ the curve approacbes the light line (LL), and for $k \rightarrow \infty$ it approaches tbe asymplotic value $\omega_{\mathrm{sp}}$ which depends on the conduction eleciron density. The dashed curve corresponds to the case of an incteased temperature

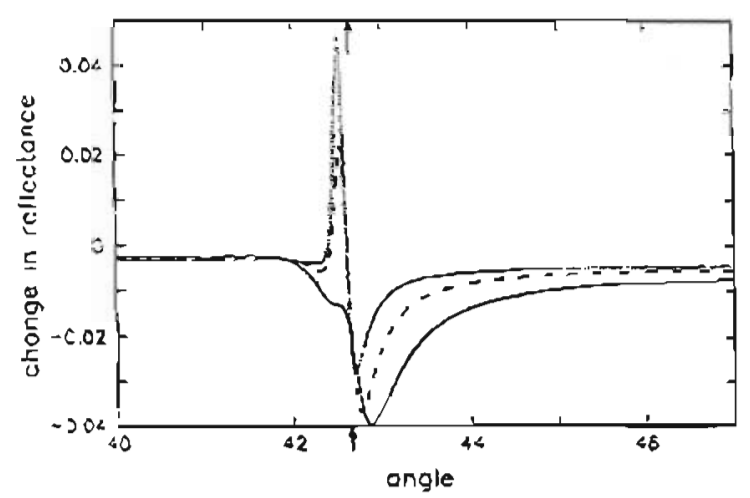

Fig. 2 Calculated thermoreflectance signal amplitudes as a function of the angle of incidence. The anrows denoce the surface plasmon resonance angle. The dashed curve corresponds to a thickness of $50 \mathrm{~nm}$, which enables complete conversion of photons into surface plasmons at the resonance angle. The dotted and the solid curve correspond to film thicknesses of $60 \mathrm{~nm}$ and $40 \mathrm{~nm}$, respectively. Calculations were periomed for a suriace temperature rise of $\Delta T=100 \mathrm{~K}$ and a light wavelength of $6328 \mathrm{~nm}$. The angle of solal reflectapoe is $41^{\circ}$

models the temperature in the film as well as at the film surface.

This change in the dielectric function is based primarily on the following two effects: on the one band, the damping of the conduction electrons is enbanced due to tbe increased number of thermal phonons. This results in an increase of the imaginary part of the dielectric function and consequently, in a broadening of the surface plasmon resonance. On the other hand, the thernal expansion of the film will reduce the electron density, thus lowering the plasma frequency of the metal, which is given by [7]

$\omega_{\mathrm{P}}^{2}=n e^{2} / \varepsilon_{0} m$

( $n$ is the density, $m$ the effective mass of the conduction electrons). This affects the dispersion relation of the surface plasmons, which is depicted scbematically in Fig. 1. Because the asymptotic frequency for $k \rightarrow \infty$, denoted as $\omega_{S P}$ in Fig. 1, is directly proportional to $\omega_{\mathrm{P}}[T]$, it is also lowered. The dashed curve shows the situation when the temperature is increased. The surface plasmon wave vector which cortesponds to the frequency $\omega_{L}$ of the detecting $c w$ laser beam is shifted to a higher value, which implies that the resonance dip is stifted to a larger angle of incidence. It is obvious from the shape of the dispersion curve that this effect strongly depends on the cw laser frequency. Particularly large changes are expected near $\omega_{L}=\omega_{\text {sp }}$. In addition to changing the dielectric function of the film material, the thermal expansion affects the resonance curve also by means of the strong dependence of the plasmon-photon coupling on the thickness of the metal film.

To evaluate the expected effects in more detail, we performed a numerical calculation employing the transfer matrix representation of Fresnel's formulae [8]. The optical constants used for the silver were obtained by a least-squares $5 t$ of the surface plasmon resonanoe which bad been measured using a Ne-Ne laser $(\lambda=632.8 \mathrm{~nm})$. Good agreement wias found with values given in the literature [9] (the imaginary part of the measured dielectric function was somewhat larger than the literature value, which is likely to be due to contamination of the film material during the evaporation process). The temperature dependence was obtained using the Drude model of a free electron plasma, which has been shown to describe well the optical properties of noble metals for visible light [9]. It should be noted at this point that for tbe calculation of the thermal expansion of the film one must not use the linear thermal expansion coefficient $x$ of the bulk material. Since the film may only expand into the normal direction, one has to employ a corrected thermal expansion coefficient $\alpha$ for the expansion of the film tbickness, which is readily shown to be given by

$\alpha^{\prime}=\alpha(1+\mu) /(\hat{\imath}-\mu)$,

where $\mu$ is Poisson's number of the film material. Since $\mu$ is usually close to 0.3 (depending on the material and its morphology), $\alpha^{\prime}$ is about twice the usual thermal expansion coefficient $\alpha$, which shows the importance of this correction. For the thermal expansion coefficient we used $\alpha=-1.87 \times 10^{-5}[10] ; \mu$ has been set to 0.3 as a typical value for silver [10].

In Fig 2, the transient thermoreflectance signal expected for a temperature rise of $100 \mathrm{~K}$ is shown for three different film thicknesses as a function of the angle of incidence. Whereas for a thickness of $40 \mathrm{~mm}$ (solid curve) the signal is negative for all angles, a "bipolar" behaviour is present for 50 non (the thickness of maximum coupling) and $60 \mathrm{~nm}$. Outside the resonance, below the total reflectance angle, one has just the usual thermoreflectance signal. Obviously, the resonance enhancement due to the surface plasmons amounts to about one order of magnitude. Performing similar calculations for shorter wavelengths, we found that the sensitivity could still be increased: at a wavelength of $400 \mathrm{~nm}$ instead of $632.8 \mathrm{~nm}$, the calculated signal amplitudes were found to be another order of magnitude larger, confirming the suggestions above. In this study, however, we want to concentrate on the He-Ne laser radiation, since this is the one used in our experiment for the excitation of the suriace plasmons.

\section{Experiment}

The experimental setup is depicted in Fig. 3. The sample was prepared by evaporating a silver film with a thick- 


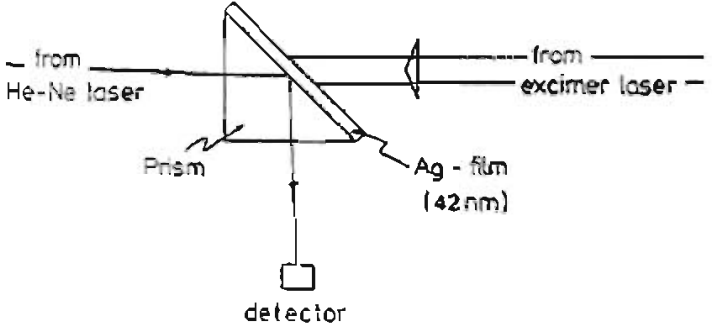

Fig. 3. Experimeotal selup. The He-Ne laser serves to excite the surface plasmons which propagate on the metal-vacuum interface. The excimer laser beam coming from the vacuum side accomaplisbes the heacing of the sample surface
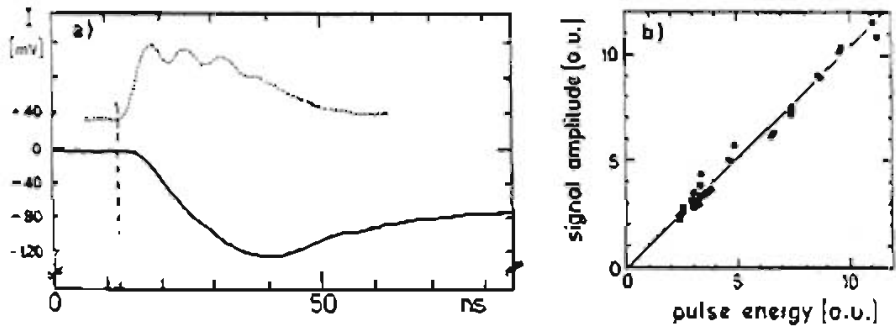

Fig. 40. Ioleasity transient taken at the surface plasmon resonance angle. The temporal laser pulse profile is show by the dotted line for comparison. The signal is obviously proportional to the integrated incoming laser power. b Signal amplitude as a function of tbe pulse ecergy. A linear bebavior is apparent, as expected for the surface remperature

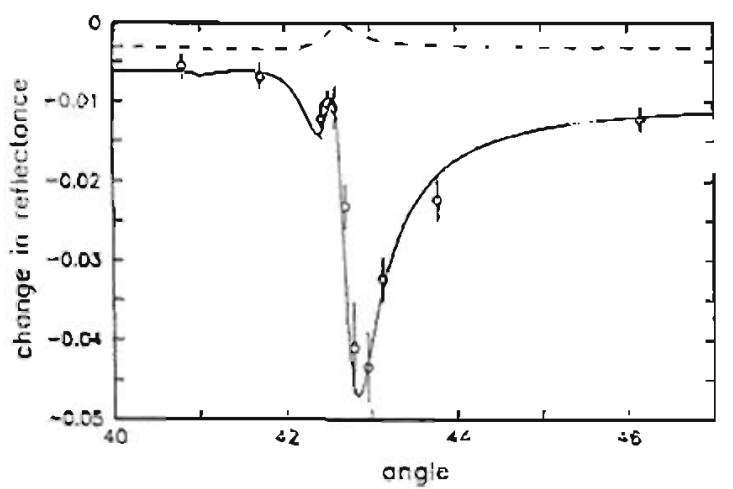

Fig. 5. Peak values of the thermorellectance signals for different angles of incidenoe. The solid line consists of the calculared signal amplitude for $\Delta \Upsilon=117 \mathrm{~K}$, plus a small correction represented by the dashed line which accounts for the diffuse scattering from pulsed laser induced indomogeneities in the sample. The error bars correspond to the slatistical fluctuations in the laser pulse energy

ness of $42 \mathrm{~nm}$ (corresponding approximately to curve $c$ in Fig. 2) onto the base of a glass prism. For heating the sample we used an excimer laser with about 20 ns pulse duration and a fuence of several $\mathrm{mJ} / \mathrm{cm}^{2}$. Its radiation with a wavelength of $248 \mathrm{~nm}$, incident onto the silver film from the vacuum side, is strongly absorbed by the silver [9], yielding short heat pulses. For the detection of the suriace plasmon resonance we used a $\mathrm{He}-\mathrm{Ne}$ laser, whose spot was small compared to (and well within) the area illuminated by the excimer laser. The reflected light intensity was measured with a fast PIN photodiode and recorded with an analog storage oscilloscope.

Figure 4a shows the signal obtained at the surface plasmon resonance angle (minimum of the reflectance curve). The temporal profile of the laser pulse is repre- sented by the dotted curve. Obviously, the surface plasmon signal is proportional to the integrated incoming laser power. This is expected when rapid energy conversion (electron-phonon relaxation) from the laser light into heat is assumed. Thus Fig. 4a demonstrates that the signal is not noticeably affected by nontbermal electronic excitation by the UV radiation of tbe excimer laser. In Fig, 4b the signal amplitude (i.e., the peak valueof the surface plasmon signal in Fig. 4a) is plotted as a function of the lasér pulse energy, demonstrating the linear relationship between these two quantities. Since the temperature rise is supposed to be proportional to the laser pulse energy to a good approximation, we can conclude that the heat induced changes in the shape of the surface plasmon resonance curve (and thus the signal) are still small enough to be considered linear-in the temperature rise.

In order ta compare the measured signal amplitudes with the theoretical prediction in more detail, we show the peak values of the thermoreflectance signals as a function of the angle of incidence in Fig. 5. The double peak structure as suggested by curve c in Fig. 2 is clearly reproduced by the data. The solid line is a numerical fit consisting basically of a calculation like those shown in Fig. 2. However, since the actual energy deposition in the sample (and thus the change in the dielectric function) may be slightly inhomogeneous due to the roughness of the silver film, we have to account for enhanced diffuse scattering from the sample in the temporal vicinity of the laser pulse. This results in an additional reduction of light intensity at the detector, proporional to the light intensity itself, i.e to the reflectivity $R(\theta)$. Therefore, to fit our data we allowed for a small correction proportional to $R(\theta)$; whieh was subtracted from the calculated signal amplitude. The result is given by the solid line in Fig. 5 . The best fit bas been obtained with a diffuse scattering correction of $-0.0035 \cdot R(\theta)$ (dashed line), a value for Poisson's number $\mu=0.30$ and a temperature rise $\Delta T=117 \mathrm{~K}$.

We were not able to measure the excimer laser Auence at the sample, but an estimate based on the laser manufacturer's specifications and the experimental geometry yields a value of $4.5 \pm 2 \mathrm{~mJ} / \mathrm{cm}^{2}$. This can be used to estimate the temperature rise at the sample surface from the optical and thermal properties of the silver film and the glass substrate. We arive at an expected temperature rise of $171 \pm 76 \mathrm{~K}$, which is consistent witb the value of $117 \mathrm{~K}$ obtained by the fit. The error bars in Fig. 5 are due to the pulse-to-pulse variation of the energy of the laser pulses. Thus, the resolution of the measurement is better represented by the width of the oscilloscope trace displayed in Fig. 3a than by the error bars. This corresponds to about $2 \mathrm{~K}$ when the angle of incidence corresponds to maximum signal. The possibility of considerable improvement conoerning the resolution is suggested by the appareat absence of noise in the signal.

\section{Conclusions}

We have shown that optically excited surlace plasmons can be used for resonance enhanced transient thermore- 
Bectance measurements of metal surface temperatures, and we recently applied this method successfully in time resolved studies of laser desorption processes [11]. In the vicinity of the surface plasmon resonanoe, the enhancement with respect to usual therworeflectance amounts to about an order of magnitude, and we mentioned that by using light with a shorter wavelength, another order of magnitude in enhancement could be achieved. The temporal resolution is limited by the thermal relaxation time of temperature gradients in the film, which is less than a nanosecond. It should be noted that the spatial resolution of this technique is limited by the decay length of the surface plasmons, which is on the order of $10 \mu \mathrm{m}$ if visible light is used for their excitation. As in all experiments employing optically excited surface plasmons, the sensitivity of the method is somewhat less for film materials other than silver. However, an enhancement in scnsitivity with respect to usual thermoreflectance is to be expected in any case.
Acknowledgements. This work bas been supporied by the Deulscle Forschungagemeinschait (SFB 2S2) and the Materialoiss. Forschungszentrum Mainz

\section{References}

1. B.M. Clemens, G.L. Eesley, C.A. Paddock: Phys. Rev. B 37, 1085 (1988)

2 A. Miklos, A. Lōrinez: J. Appl Phys 63, 2391 (1988)

3. E Kretschmann, H. Raether: Z Naturforsch A 23, 2135 (1968)

4. H. Raebber: In Physics of Thin Films, cd. by G. Hass, M.H. Francombe, R.W. Hollmann (Academic, New York 1977) VoL 9

5. Y. Levy, M. Jurich. J.D. Swaled: J. Appl. Phys. 57, 2601 (1985)

6. M. van Exter, A. Lagendijk: Phys. Rev, Lett. 60, 49 (1988)

7. N.W. Asbcroft, N.D. Mermin: Solid State Physics (HoltSaunders, Tokyo 1981)

8. M. Bora, E. Woll: Priaciples or Optics (Pergamon, Oxford 1970)

9. P.B. Jobnson, R.W. Christy: Phys. Rcv. B 6, 4370 (1972)

10. O. Loebich: In Landolt-Bomslein, ed. by H. Borchers, E. Schmitt, 6th edn. (Springer, Berlin, Heidelberg 1964) Vol. TV/2b, p. 576

11. S. Herminghaus: Thesis, Mainz 1989 